\title{
REAL-TIME EARTHQUAKE MONITORING WITH SPATIO-TEMPORAL FIELDS
}

\author{
J.C. Whittier, Silvia Nittel, Iranga Subasinghe \\ School of Computing and Information Science, The University of Maine, Orono, ME 04469, USA \\ silvia.nittel@maine.edu
}

KEY WORDS: Data Stream, Data Stream Processing, Real-time Event Detection, Earthquake Event Detection, Apache Spark, Fields, Temporal Field, Fields as ADTs

\begin{abstract}
:
With live streaming sensors and sensor networks, increasingly large numbers of individual sensors are deployed in physical space. Sensor data streams are a fundamentally novel mechanism to deliver observations to information systems. They enable us to represent spatio-temporal continuous phenomena such as radiation accidents, toxic plumes, or earthquakes almost as instantaneously as they happen in the real world. Sensor data streams discretely sample an earthquake, while the earthquake is continuous over space and time. Programmers attempting to integrate many streams to analyze earthquake activity and scope need to write code to integrate potentially very large sets of asynchronously sampled, concurrent streams in tedious application code. In previous work, we proposed the field stream data model (Liang et al., 2016) for data stream engines. Abstracting the stream of an individual sensor as a temporal field, the field represents the Earth's movement at the sensor position as continuous. This simplifies analysis across many sensors significantly. In this paper, we undertake a feasibility study of using the field stream model and the open source Data Stream Engine (DSE) Apache Spark(Apache Spark, 2017) to implement a real-time earthquake event detection with a subset of the 250 GPS sensor data streams of the Southern California Integrated GPS Network (SCIGN). The field-based real-time stream queries compute maximum displacement values over the latest query window of each stream, and related spatially neighboring streams to identify earthquake events and their extent. Further, we correlated the detected events with an USGS earthquake event feed. The query results are visualized in real-time.
\end{abstract}

\section{INTRODUCTION}

Major earthquakes have significant impact on human life and built infrastructure. In recent years several devastating earthquakes took place with significant damage such as the earthquake and tsunami in Japan (2011) and the earthquakes in Nepal (2015), Italy (2016), and New Zealand (2011 and 2016). Earthquakes are created through the constant movement of the Earth's tectonic plates and the resulting friction that is created. Early earthquake detection and wide-spread early warning systems are essential to shut down infrastructure such as gas, chemical or nuclear power plants and transportation, and save human life. Earthquakes can be detected by two characteristic waves, the P-wave and the Swave (Bullen and Bolt, 1985). The $\mathrm{P}$ wave travels much faster than the $\mathrm{S}$ wave, and therefore provides a longer time window for early warning. The S-wave indicates the start of the significant movements of the earthquake itself.

Today, most physical sensor systems to detect and measure earthquakes are run by government agencies. Measurement stations with expensive and accurate equipment are deployed and the data is analyzed by seismology scientists; this data is often not available to the general public in its raw form. However, in recent years several approaches have been explored to use alternative, inexpensive technology such as commercial-off-the-shelf GPS sensors to detect movement along earthquake faults (Grapenthin et al., n.d. Grapenthin et al., 2014. Wright et al., 2012, Clayton et al., 2015 Hudnut et al., 2002). These GPS based sensor networks measure displacement, and send real-time data measurement streams. Other efforts include using smartphone vibration sensors for earthquake detection (Panizzi, 2016, Kong et al., 2015). We can reasonably expect that in the future, an increasing number of up to potentially thousands of sensors will be

\footnotetext{
${ }^{*}$ Corresponding author
}

deployed in earthquake-prone regions, from smartphones to inexpensive GPS stations, which continuously measure and report the Earth's movement in real-time. Such sensor networks will produce high throughput data streams of thousands of updates/sec that need to be analyzed in real-time.

While an earthquake is a continuous movement of the Earth in space and over time, measurement station provide only discrete samples of this movement. Each sensor only observes the event at a specific, local position, and all observations need to be integrated over space and time to assess the scope and magnitude of an earthquake. This synthesis across potentially thousands of streams should be done in near-real-time (i.e. ca. 1-2 second delay). Programming this task is no small feat using conventional technology, such as databases or programming languages with standard libraries.

In previous work, we proposed the Field Stream Data Model (Liang et al., 2016), which builds the foundation for modeling and querying spatio-temporal continuous phenomena in DSE A temporal field represents a high-level abstraction of the temporal continuity of a phenomenon and hides the sampling characteristics of a sensor. The user accesses a temporal field as if temporally continuous. Any request to the temporal field (programming abstraction) for a value at any particular time point of the field's temporal domain is computed in real-time and returns either a measured or an estimated value for that time instant. Generic (non spatial) DSE, which have been introduced about a decade ago, facilitate the high throughput query processing for very large numbers of real-time data streams. For instance, compared to Database Management System (DBMS) technology with a throughput of 500 updates/s(Stonebraker et al., 2005), DSE scale up to 1.5 Mio update/s per CPU core(Carbone et al., 2015). DSE simplify the programming of analysis tasks over streams by 
providing a query language, which consists of a library of customizable functions such as filter(), map(), reduce() and others.

In this paper, we pursue two objectives: first, we study the feasibility of our previously proposed field model abstractions by applying them to a real world problem, that is real-time earthquake event detection with sensor data streams. Secondly, we explore the implications of a practical implementation integrating the field data types into a commercial or open source DSE For this study, we chose the open source system DSE Apache Spark (Apache Spark, 2017). In detail, we modeled the problem of earthquake detection as a problem of processing continuous fields. Our input data are 250 real-time sensor streams of the Southern California Integrated GPS Network (SCIGN), here, each a sensor generates 1 update/s. An earthquake can be seen as a spatio-temporal event that can be extract from an underlying spatio-temporal field. The underlying field is the continuous movement of the tectonic plates, which is measured by the network of GPS stations. The movement captured by a single sensor is represented as a temporal field over this sensor. The temporal fields of all sensors of the SCIGN are seen as a spatiotemporal field since spatially neighboring sensor stations measure different, but related aspects of the same earthquake. Thus, an earthquake can be captured by first analyzing the temporal fields over sensors. Once analysis of the temporal fields indicates above threshold movement values, the temporal fields are spatially correlated, and the start, end and spatial scope of an earthquake event can be deduced. We implemented this approach within Apache Spark as continuously executed Apache jobs and the results are visualized in real-time.

The remainder of this article is organized as follows: in Section 2, we discuss the background of this work, including earthquake detection in Southern California and the concept of abstracting sensor data streams as temporal fields. In Section 3, we state our objectives for this work. Section 4 and 5 describe our approach, that is the mapping of streams to field Resilient Distributed Datasets (RDDs) and the implementation of real-time earthquake detecting using Spark queries. Section 6 discusses our lessons learned and our conclusions follow in Section 7.

\section{BACKGROUND}

\subsection{Monitoring Earthquakes in Southern California}

Earthquakes take place due to the movement of tectonic plates, and the tension between them, while moving in different directions. The type of movement depends on the geographic location of a tectonic plate and its connecting plates. In this paper, we are interested in the San Andreas Fault in Southern California. The San Andreas Fault is a fracture line between the Pacific Plate that moves in the North direction and the North American plate, which moves South, and both plates sitting slightly on top of each other. Friction between both plates builds up tension over time, which releases via an earthquake. The movement is relatively slow, and it can take decades for significant tension to build up. Thus, displacement along the known smaller and larger fault lines is of most interest.

The SCIGN (Hudnut et al., 2002) consists of roughly 250 Global Positioning System (GPS) stations, distributed throughout Southern California with an emphasis on the greater Los Angeles metropolitan region. The major objective of the SCIGN network is to provide regional coverage for estimating earthquake potential throughout Southern California, and to learn more about the mechanical properties of earthquake faults.

The GPS sensors are deployed in proximity to small and large faults. Each station produces an update at a rate of once per second including the latest measured position of the GPS the delta of displacement from the previous measured location in $(x, y, z)$, and error data, all available in GeoJSON format (GeoJSON, 2017). An example update message from a sensor is provided in Listing 1 The 'shaking' of an earthquake delivers displacement values (deltas) of increasing and then decreasing value over time periods. Likely, they are observable in spatially nearby stations also.

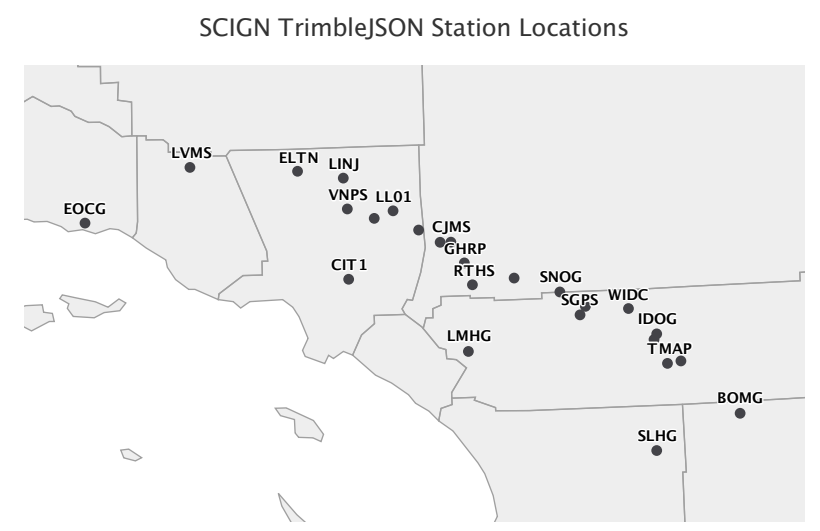

Figure 1. Locations of SCIGN sensor sites in the TrimbleJSON feed

\subsection{Real-time Sensor Data Stream Processing}

With up to 250 stations, and likely more stations in the future, the data processing of all streams in real-time becomes a programming challenge. Over the last decade, DSE have become commercially available; the Apache Foundation offers an open source DSE Apache Spark (Apache Spark, 2017). DSE are similar to DBMSs in that they offer a data model and query language, which make it significantly easier for users to program applications that require real-time processing of data streams. A user defines the stream schema and can program SQL-like queries over the streams to perform basic and complex aggregation and analysis tasks. Stream queries are expressed in terms of temporal windows over streams. For instance, a query will collect the data of the last minute, perform the query and produce a result. The query is automatically re-evaluated over the next window of arriving data from the stream (i.e. continuous query) (Chen et al., 2000). DSEs provide high throughput query processing for data streams by buffering and processing streams in main memory. Stream queries are implemented as scalable, one-pass (Muthukrishnan, 2005) stream operators; they can be parallelized automatically (Galić, 2016). Today, open source and commercial DSEs such as Apache Spark (Apache Spark, 2017) achieve query performance over streams with a throughput of $>1$ Million updates/s per CPU core(Carbone et al., 2015).

\subsection{Temporal Fields as Stream Data Types}

In geographic information science, the term field (more precisely geo field) is widely used to describe entities in physical space that are continuous (Couclelis, 1992, Kemp, 1996). A field can be continuous in space, in time or in space and time (Liang et 
al., 2016). For instance, a field implies a continuous quality of an observed phenomenon in the real-world, such as temperature, that is present at every point in time and space within a defined region on Earth. Similarly, an earthquake can be considered a spatio-temporal event that is extracted from a continuous spatiotemporal field. Here, the underlying spatio-temporal field can be the change in position of each spatial (point) location on Earth between two time instances $t_{i}$ and $t_{j}$. To compare movements for multiple time instants, $t_{0}, t_{1}, \ldots, t_{n}$, rather than calculating the difference between the position at $t_{i}$ with that at $t_{i-1}$, using a static reference position and calculating the difference between the reference position and the position at $t_{i}$ simplifies the analysis.

Today, scientists mainly deal with spatial fields; many different computer representations are common (raster, TINs, isolines, etc). While implementations of these computer representations enable specialized analytical operations in geographic information systems, the diversity and lack of coherence of representation hinders convenient integration and cross-cutting analyses (Kemp, 1996). This problem is exacerbated if the continuity over time is also considered such as with the need to integrate sensor data streams (Liang et al., 2016).

In (Liang et al., 2016), we introduced fields as Abstract Data Types (ADTs) for stream data models. The core idea is that sensor data streams are abstracted as temporal fields, spatial fields or spatio-temporal fields, depending on the application needs. Fields are approximated based on observations, so-called sampled fields, and a field ADT implementation consists of the observations and an approximation function that uses the observations to estimate all the values at points at which the field was not directly sampled. Thus, the field ADT 'behaves' as if the temporal field is continuous, and automatically approximates the continuity of the values over time based on the observations. For more details and the formal definitions of fields, please see (Liang et al., 2016).

\section{OBJECTIVES}

In this paper, our objective is to assess the feasibility of Field ADTs as well as DSEs for a real-world problems, in this case realtime earthquake monitoring. We identified publicly available live data streams, and selected the SCIGN network. The streams are accessible via sockets in near-real-time. [SCIGN delivers 'raw' GPS values that we cast to temporal fields. The temporal fields we aggregated over time and space to detect events. Additionally, we used a United States Geological Survey (USGS) feed reporting officially reported earthquake events, with the purpose of comparing our detected events with the USGS events.

\subsection{Data sources}

3.1.1 SCIGN The Southern California Integrated GPS Network SCIGN) consists of roughly 250 GPS stations located in Southern California (Hudnut et al., 2002). Each station produces an update at a rate of once per second including a time stamp, the latest spatial position, delta to a reference position, and error data in GeoJSON format. Additionally, a topic (a kind of composite type), in the format

[ProcessingEngine].[Region].[SiteCode].[SolutionType], is included in the GeoJSON document that identifies the lineage of the data. A real-time client is available through SCIGN to connect to a live GeoJSON stream for topics (customized subsets) that meet the wildcard query topic. At the time of this article, only the TrimbleJSON processing engine feed included computed deltas in the delta feature. The 32 sites in the TrimbleJSON feed are depicted in Figure 1 and an example message from SCIGN is shown in Listing 1]

Listing 1. A TrimbleJSON Message from SCIGN

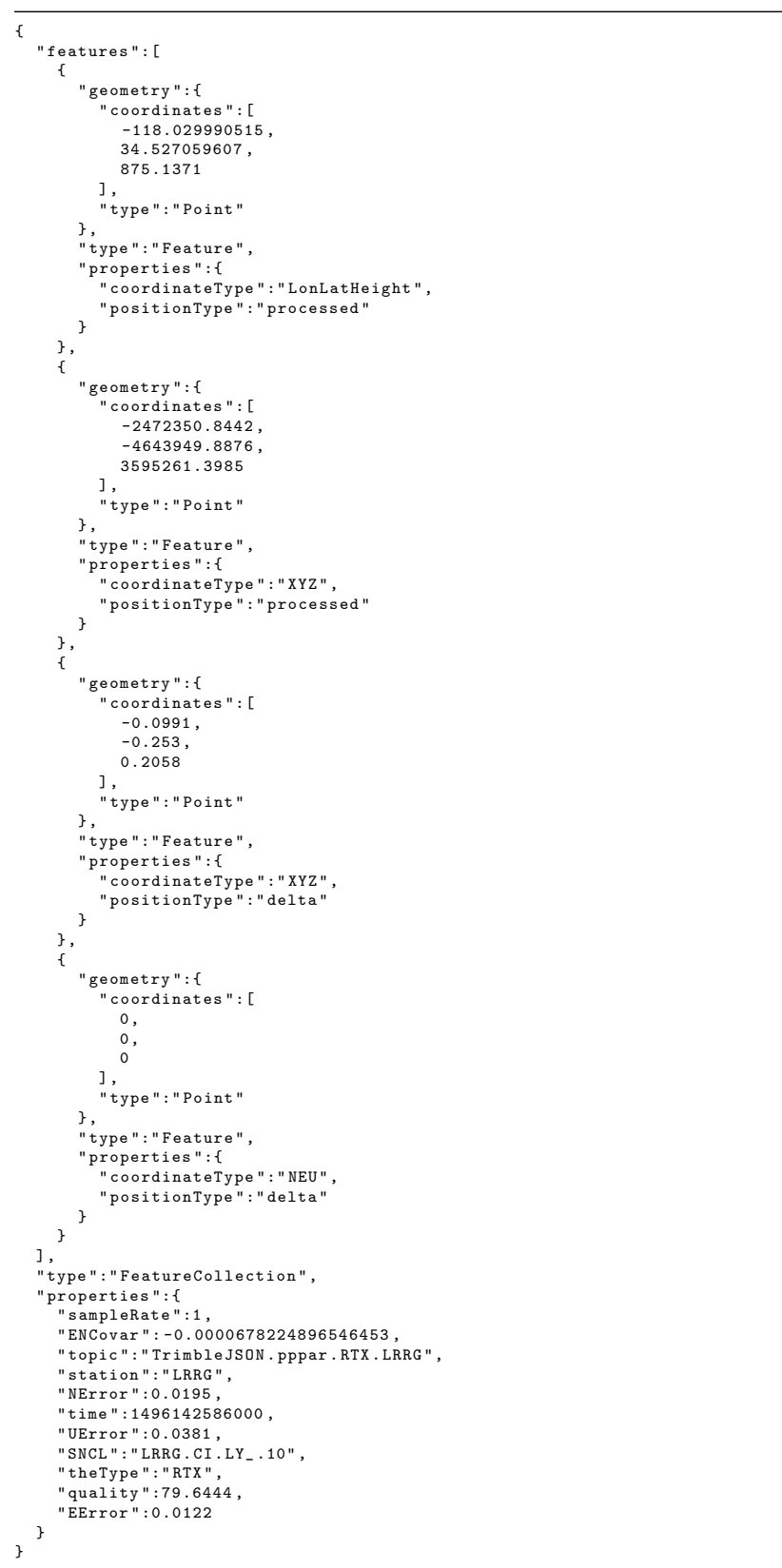

3.1.2 USGS The USGS provides "real-time" feeds and notifications for earthquake events (Real-time Feeds and Notifications, 2017). Though the feeds are referred to as "real-time," there is a delay between the origin time of the event, when the earthquake began, and the updated time, when the event is published to the GeoJSON feed, which is over 20 minutes. The GeoJSON feed URL supports user provided parameters including filtering events by spatial location, origin time, and update time. To retrieve new events detected by the USGS within a bounding box of interest, a user polls the feed for updates since the time of the last update. An example event is shown in Listing 2

Listing 2. A Feature from the USGS Earthquake Feed 


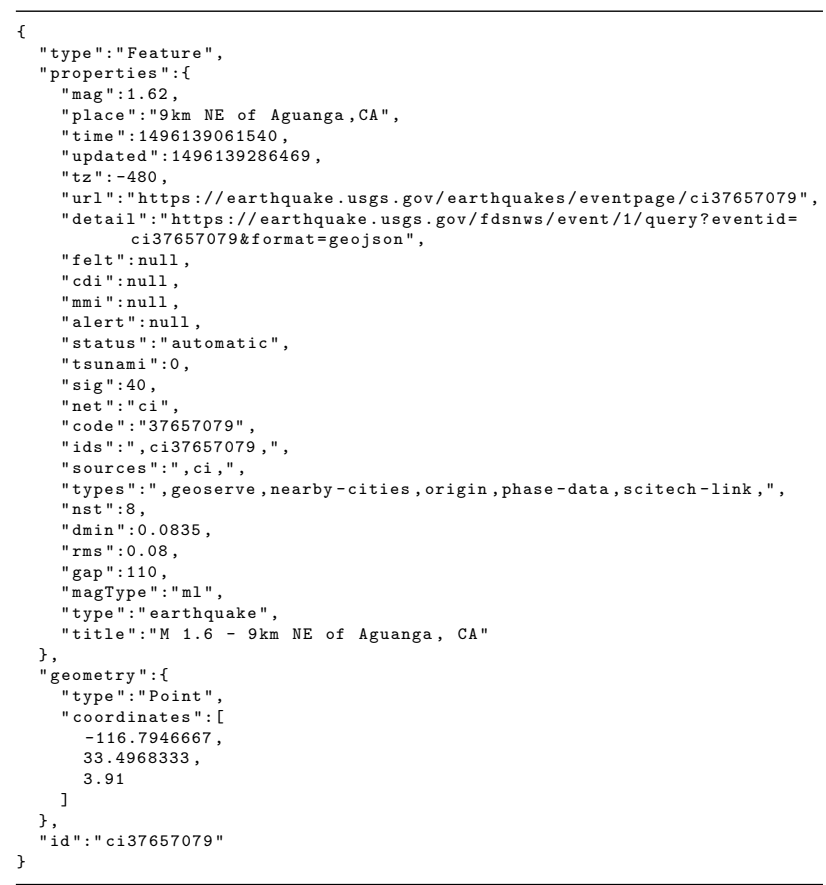

\subsection{System Components and Architecture}

Our real-time earthquake detection system is built using using mostly open source software components that are loosely coupled in a micro service architecture to enable system scalability.

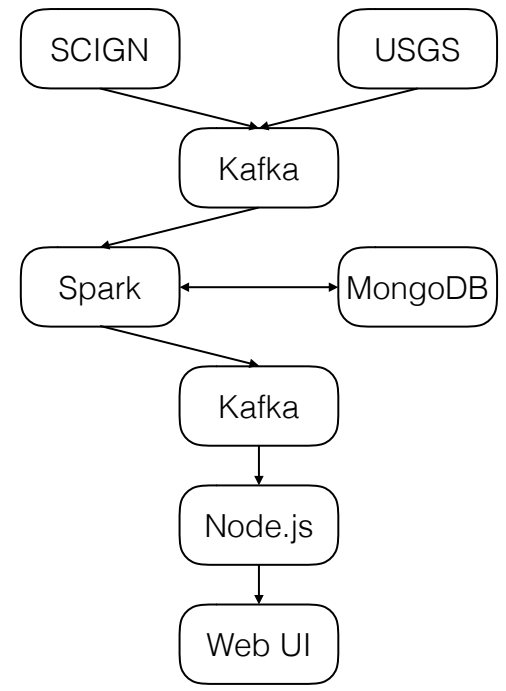

Figure 2. System Components and Data Flow

For the implementation, we chose Apache Spark (Apache Spark, 2017). Spark is used for expressing and processing queries over streams. Streams can be be generated by data sources or by queries, i.e. their continuously delivered results over windows. A stream query is both a stream consumer and a stream producer (of result tuples). Since the rate of produced and consumed tuples needs to be carefully orchestrated, we use Kafka (Apache Kafka, 2017) for message passing between stream inputs, processing components, and output consumers. Capped collections in MongoDB are used to cache the SCIGN stream, so that when delayed USGS events arrive, the USGS event and time series of nearby SCIGN stations can be combined.
3.2.1 Apache Spark: Stream Processor Apache Spark (Apache Spark, 2017) is an open source cluster computing platform supporting in-memory processing of stored and streaming data in the form of batch and streaming jobs, respectively. For stream processing, Spark partitions a stream into windows, called a discretized stream or DStream, and processes the data in Spark using a program written in Scala, Java, or Python.

3.2.2 Apache Kafka: Message Broker Apache Kafka (Apache Kafka, 2017) is a distributed, massively scalable message broker designed for real-time streaming applications. Kafka acts as a centralized message broker, passing data between input data streams and and stream processing queries, between stream processing queries, and between stream processing queries and other consumers. Kafka utilizes topics that producers publish to and consumers subscribe to.

3.2.3 MongoDB: Data Storage MongoDB(MongoDB, 2017) is a open source document-oriented database program and classified as a NoSQL database system. MongoDB uses JSON-like documents with schemas. MongoDB supports AdHoc queries, indexing and real-time aggregation; this provides powerful tools to access and analyze data with high throughput. MongoDB supports capped collections, which are fixed-size collections that support high throughput operations. These collections work in a way similar to circular buffers. In general, MongoDB collections can be used as a persistent storage for historical data analysis.

How the data streams are consumed, analyzed, and fed to a live dashboard is discussed further in the following sections.

\section{MAPPING GPS STREAMS TO TEMPORAL FIELDS}

In Section 1. we described the data format of SCIGN updates as they arrive. The stream tuples are formatted in GeoJSON by SCIGN and Spark is able to parse and process JSON formats directly, i.e. operators are available to extract the schema and the tuples. In Spark, the basic data structure to store and process data is an RDD An RDD is a immutable, read-only, logical data collection that can contain any type of Python, Java, or Scala objects, including user-defined classes. Transparently to users, Spark divides a RDD into logical partitions, which may be processed on different nodes of a cluster in parallel.

The implementation of a Temporal Field type consists of two components: the observation data and an interpolation function (or a set of different interpolation functions for flexibility). To provide values at time instants of the temporal field at which the field has not be sampled directly the field implementation approximates the requested value based on both components. Thus, any programming tasks using Field types can abstract from the notion of observations, and work based on the assumption of continuity.

To implement a Temporal Field type in Spark, we programmed an operator that extracts relevant data from the incoming SCIGN observation feed and creates a Field $R D D$ The Field RDD is a generic, flexible schema for spatio-temporal data streams that supports both sensor observations and spatio-temporal events. It has a fixed schema for observations, which is defined as

$$
<\text { id }><\text { location }><\text { time }><\text { value }>
$$

- The $\langle i d\rangle$ is an identifier to describe the other attributes, for example a sensor location code (e.g., TrimbleJ- 
SON.pppar.RTX.7ODM) or event id. An id can be a string, number, or null.

- The $<$ location $>$ is the location that the value corresponds to. A location can be point, line sting, polygon, or null. A point consists of a (latitude, longitude) pair.

- The $<$ time $>$ is the time the value was collected/sampled A time can be an instant or interval; currently, only instants are considered.

- The $\langle$ value $>$ is either null or a valid JSON string. This string can include nested structures or be flattened depending on the limitations of the implementation environment.

Any Sparkqueries are expressed using operators such as filter, map, and reduce, which are provided by Spark. Once the data stream is transformed into a field RDD field operators to analyze the streams can be written. The Spark query results are stored as an RDD again. The details of the field analysis operators are detailed in the following section.

\section{REAL-TIME EARTHQUAKE EVENT DETECTION}

We approach detecting earthquake events in two steps: a realtime event detection step using live GPS streams and a response time of a few seconds, and a post-mortem event detection step using USGS earthquake event notifications and comparing such an event to our detected event(s) and the raw sensor data streams that we cached for this purpose. All tasks are automated, and run continuously. Results are visualized in real-time in web application, which can be found at (Real-time Earthquake Monitoring Visualization for SCIGN Stations, University of Maine, 2017).

\subsection{Real-time Event Detection using GPS Streams}

5.1.1 SCIGN Stream Processing Pipeline The GPS streams from SCIGN are analyzed and visualized to extract earthquake events. At a high level, the data flow follows the system architecture presented in Figure 2

In detail, the data flow begins with a Node.js client, provided by SCIGN to consume the live stream of data hosted by SCIGN The client uses Socket.IO (Socket.IO, 2017) to pass the desired SCIGN topics back to the data source to customize the data that should appear in the stream. To get all data from the TrimbleJSON processing engine, the topic is specified as TrimbleJSON .*.*.*. Socket.IO is then used to stream all tuples with the TrimbleJSON processing engine to the client. The output from the SCIGN client is piped to a Kafka console producer, which loads the messages in the SCIGN stream into a scign-flattrimble Kafka topic. Note, that the stream is called a series of messages, and each update is a message.

Once a message is loaded into Kafka, it is available to be processed by Spark. The Spark stream processing jobs subscribe to and read from an input topic, perform some data transformation, and then publish the results back to Kafka as a new stream to a different topic. This new stream can be consumed by another Spark job or the Node.js server for the visualization. We use MongoDB for data storage, with some jobs (queries) writing to or reading from MongoDB. During processing, the stream is grouped by the attribute sensor identifier. For each sensor, a window is defined, and the observations within the window are aggregated. An overview of the Spark streaming job can be found below.
The SCIGN Trimble Consumer Spark job feeds raw SCIGN data to the web visualization by listening to the scign-flat-trimble Kafka topic, partitioning the stream into time-based windows, transforming the output to the desired format and publishing the transformed messages to the scign-raw-output Kafka topic. It also performs aggregation to calculate the range of the $x, y, z$ deltas and a combined 3D delta for each sensor [SCIGN topic) per window. The deltas (for $x, y$, and $z$ ) from each observation are aggregated individually using Equation 1 to calculate their range and together using Equation 2 to calculate a combined 3D range.

As described further in Section 5.1.3, the Node.js server subscribes to this topic for visualizing the data.

$$
\begin{array}{r}
\operatorname{range}\left(\Delta_{x}\right)=\max \left(\Delta_{x}\right)-\min \left(\Delta_{x}\right) \\
\sqrt{\left(\operatorname{range}\left(\Delta_{x}\right)\right)^{2}+\left(\operatorname{range}\left(\Delta_{y}\right)\right)^{2}+\left(\operatorname{range}\left(\Delta_{z}\right)\right)^{2}}
\end{array}
$$

Following, the aggregated stream is published to the scign-aggoutput topic.

5.1.2 Filtering Events from Aggregated SCIGN Data A simple specification of an earthquake event is defined as an occurrence of

$$
\operatorname{range}_{\triangle 3 D}>T
$$

where $T$ is a threshold value. In order to extract events, a filter job has been implemented that filters the output of the scign-aggoutput Kafka topic to select all aggregated tuples whose 3D delta exceeds the threshold value. The choice of the threshold value is discussed in Section6 The threshold is supplied as an argument when the Spark job is submitted; the job is described in detail below.

1. SCIGN Event Filter Spark job listens to the scign-aggoutput Kafka topic, which applies a filter to find the aggregated SCIGN] events above the provided delta threshold. The filtered aggregated SCIGN events are published to scign-filter Kafka topic to be consumed by another Spark job, which stores the detected events in the scignevent-archive collection.

2. SCIGN Event Extractor Spark job that processes detected events retrieves the raw SCIGN data from the MongoDB capped collection scign-raw, for the sensor that detected the event and its $k-1$ nearest neighbor sensors within $r$, and within a specified time window around the event. The data about the event, and the raw data from the $k$ sensors are combined and stored in the scign-event-archive collection and output to the in-scign-model-event Kafka topic.

3. The in-scign-model-event Kafka topic is then consumed by the SCIGN Model Transformer Spark job to transform the messages to the desired format for the visualization and it published the data to the scign-event-output Kafka topic.

5.1.3 Web Visualization Several Spark job query results are published in real-time to a web-based visualization client. Behind the web visualization are two servers. Static content is served using the open source NGINX web server and dynamic data from 


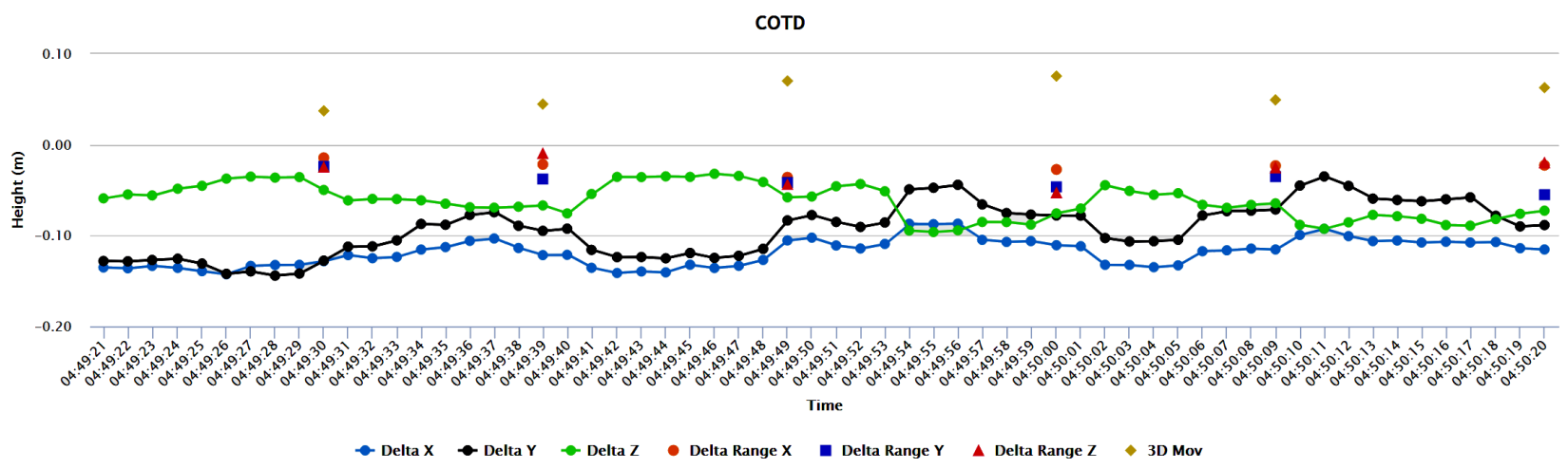

Figure 3. Real-time raw delta $x, y, z$ and aggregated ranges for a SCIGN sensor site

the Spark system is served using Node.js, an open source, serverside JavaScript runtime environment. Node.js subscribes to several Kafka topics (scign-raw-output, scign-agg-output, scignevent-output and usgs-event-output) in order to push the real-time data and events to the web-based visualization client. Data is streamed from Node.js to the web-based visualization client using the Socket.IO (Socket.IO, 2017) JavaScript library. The webbased visualization client then loads the real-time data into time series graphs generated using the Highcharts JavaScript library (Highcharts, 2017).

In Figure 4 the raw and aggregated streams are visualized on the right of the dashboard, while the left side shows the map of sensor site and their aggregated 3D deltas. The web-based map is built using the Leaflet (Agafonkin, 2017) JavaScript library that visualizes the aggregated $3 \mathrm{D}$ delta range and events. A circle is used to visualize the aggregated 3D delta range for each sensor site, where the relative diameter of the circle is proportional to the aggregated 3D delta value. The color of the circle is red when the aggregated 3D delta value at a site exceeds a threshold, otherwise it is black.

\subsection{Correlating GPS Streams with USGS Events}

The second, alternative approach for earthquake analysis uses a feed publishing earthquake events occurring in California, provided by USGS Each event in this feed is extracted and we visualize the time series data from the $k$ nearest SCIGN sensors around the reported earthquake and within a time window around the occurrence of the USGS reported event. This approach for processing the USGS events extends the SCIGN processing approach mentioned in subsection 5.1

5.2.1 USGS Stream Processing Pipeline A stand-alone Python program consumes the parameterized USGS earthquake event within a bounding box surrounding California. In order to only fetch the latest event(s), the feed is polled periodically for event updates and compares the last updated time-stamp with the most recent event update that we have processed. The last updated time-stamp is fetched from the usgs-raw-archive MongoDB collection which is used to store USGS events locally. The consumed stream from USGS is then published into the inusgs-raw Kafka topic and serves as input to the Spark processing pipeline.

1. The USGSEvent Transformer Spark job consumes from the in-usgs-raw Kafka topic and publishes the events it receives to the output topics out-usgs-event and out-usgs-raw.

2. The USGS Consumer Spark job listens to the USGS earthquake events in the out-usgs-raw topic and stores the events in the MongoDB collection usgs-raw-archive to make them available for further analysis.

This processing pipeline prepares the USGS events to be combined with the raw SCIGN data.

\subsubsection{Combining USGS Events and SCIGN Observations}

1. The USGSEvent Extractor Spark job processes the USGS earthquake events in the out-usgs-event topic and selects the set of $k$ nearest stations within a radius of $r$ miles around the reported earthquake location contained in a USGS event. For this set of stations, the raw data within a predefined time window, relative to the time (origin time) of the USGS earthquake event, are fetched from the MongoDB capped collection scign-raw. The data about the USGS event, and the raw data from the $k$ sensors are combined and stored in the usgs-event-archive collection and output to the in-usgsmodel-event Kafka topic.

2. The in-usgs-model-event Kafka topic is then consumed by the USGS Model Transformer Spark job to transform the messages to the desired format for the visualization and published to the usgs-event-output Kafka topic.

\section{DISCUSSION}

Earthquake detection: We developed a framework for real-time earthquake detection, which is still under development. We simplified our approach of querying the SCIGN data, and we do not claim that this approach is as advanced as that of a seismology domain expert. This leads to a second question: is a threshold on the range of deltas from the SCIGN network sufficient to detect earthquake events and at what magnitude sensitivity? We experimented with the SCIGN data over the last 3 months, and during this time period no significant earthquakes occurred, which limited our experience with reference data. In order to classify deltas in 5.1.1 and correlate them to earthquake events with different magnitude, we selected a GPS threshold that was reported in the literature in (Grapenthin et al., 2014), an operational system using GPS data for earthquake detection. However, this threshold 


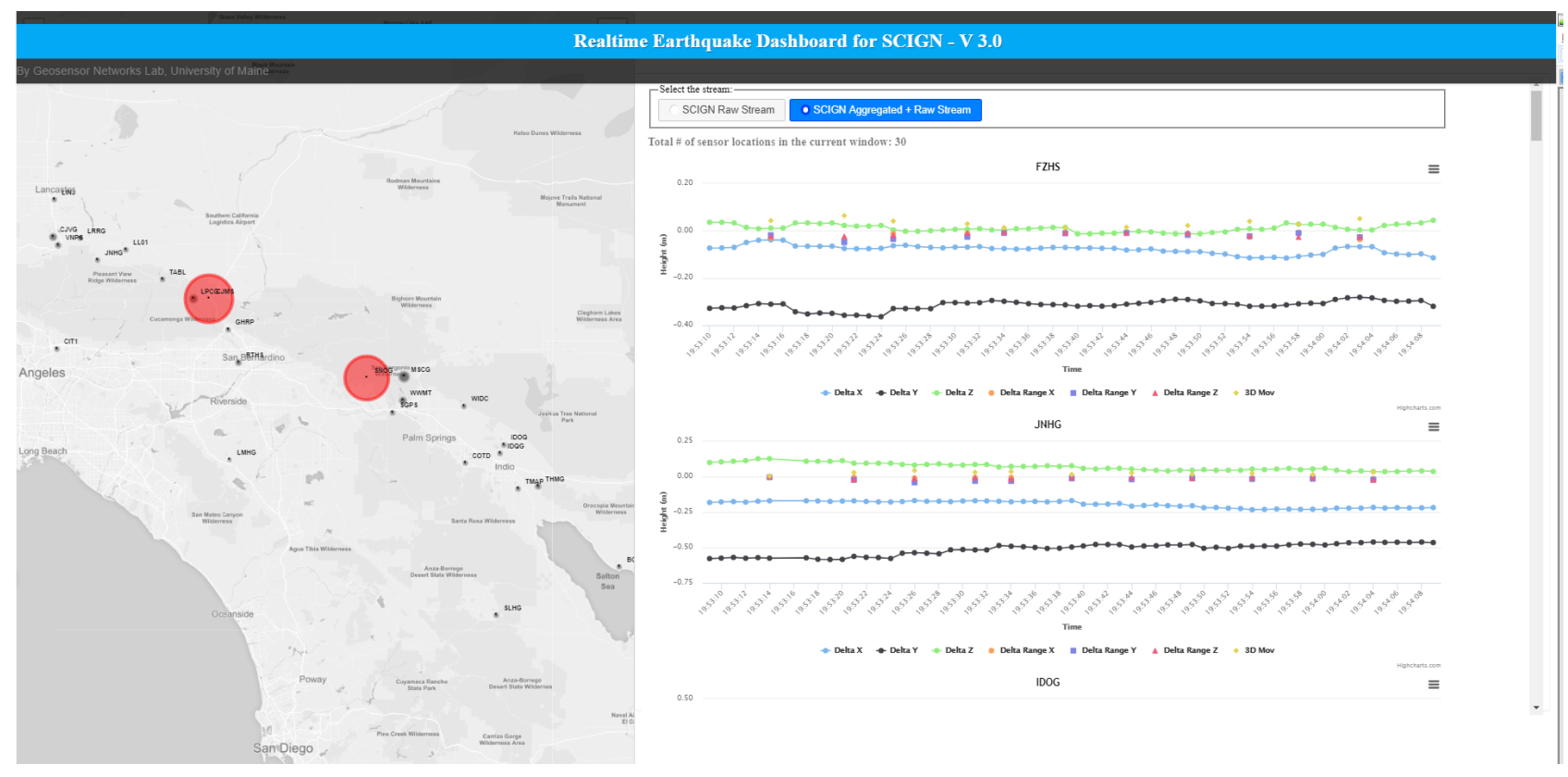

Figure 4. Web Visualization Client Dashboard with Spatial Visualization and Live Streams.

relates to earthquakes of magnitude 7.0. It is currently unclear how accurately smaller earthquakes can be detected using the currently deployed GPS devices. However, we believe that question is likely to be resolved by future technology development.

Experimenting with parameters: The system was designed to be able to test the impact of different parameters. The radius, $r$, and number of neighboring sensors, $k$, around an event are two parameters for combining events detected from the SCIGN] stream and in the USGS feed with data streamed by SCIGN It is important to note that although both approaches return $k$ sensors within $r$ around an event, the locations of an event in the SCIGNEvent Filter are the locations of SCIGN sensors where as in the USGS feed the location is where the earthquake originated. This means that if the SCIGNEvent Filter and the USGS both detect the same event, the location of the detected event will be different and thus the $k$ nearest sensors to each event location are not necessarily the same. An additional consideration is whether the choice of $r$ and $k$ depend on the magnitude of the earthquake event and whether these parameters should be fixed or adaptive.

Additional parameters relate to bounds of time-based query windows used for aggregation and visualization. Currently, for aggregating the SCIGN stream, a tumbling window of $5 \mathrm{~s}$ is used, meaning that data will be partitioned into non-overlapping $5 \mathrm{~s}$ windows. In Spark overlapping sliding windows are also supported, which could be configured to both increase the time interval and shorten the time between result updates of a query. For combing events, from the SCIGN stream and the USGS feed, the interval of the SCIGN streams to be extracted needs to be specified. This interval should be large enough to capture the behavior of the sensors during an event.

Scalability: By using a system architecture with loosely coupled components, individual components of the architecture can be scaled out to meet processing and storage requirements. Additionally, the system was designed so that it can be deployed using a microservice architecture, where each component could be elastically scaled or new code could be deployed to a component while the entire system continued to operate. For example, in Spark where most of the data processing is done, each Spark job can be managed independently as the Spark jobs are integrated using Kafka as a message broker.

Field Library: First, we explored the design to implement a sensor stream as a temporal field within an open source DSE Here, the main lesson learnt is that support for a field 'type' is less likely to be a data type implementation. Instead we envision that spatiotemporal field support is implemented as a field RDD type and library of field functions that interface with Spark functions and alleviated coding needs for programmers.

\section{CONCLUSION}

In this paper, we presented a feasibility study of using the field stream data model (Liang et al., 2016) and an open source DSE, Apache Spark (Apache Spark, 2017), for real-time earthquake event detecting using the GPS streams of SCIGN We presented two approaches to earthquake detection; in the first approach, we processed the sensor data streams using window stream queries, and found the maximum displacement in each window. These values were filtered against a threshold value, and events were identified. We stored raw data temporarily in MongoDB to support the second approach, in which we pulled the latest earthquake notifications from USGS. and correlated the reported events with our detected events and the raw data. All queries analyze the latest data updates continuously and events are reported within 1-2 seconds after the first indication. Real-time data visualization is available at (Real-time Earthquake Monitoring Visualization for SCIGN Stations, University of Maine, 2017).

\section{ACKNOWLEDGEMENTS}

The authors would like to acknowledge the National Science Foundation for supporting this work via Award No. 1527504.

\section{REFERENCES}

Agafonkin, V., 2017. Leaflet: an open-source javascript library for mobile-friendly interactive maps. http://leafletjs.com (8 July 2017). 
Apache Kafka, 2017. http://kafka.apache.org (24 May 2017).

Apache Spark, 2017. http://spark.apache.org (24 May 2017).

Bullen, K. E. and Bolt, B. A., 1985. An Introduction to the Theory of Seismology, 4th Ed. Press Syndicate of the University of Cambridge, Cambridge.

Carbone, P., Katsifodimos, A., Ewen, S., Markl, V., Haridi, S. and Tzoumas, K., 2015. Apache flink: Stream and batch processing in a single engine. Bulletin of the IEEE Computer Society Technical Committee on Data Engineering.

Chen, J., DeWitt, D. J., Tian, F. and Wang, Y., 2000. NiagaraCQ: A Scalable Continuous Query System for Internet Databases. In: Proceedings of the 2000 ACM SIGMOD International Conference on Management of Data, SIGMOD '00, ACM, New York, NY, USA, pp. 379-390.

Clayton, R. W., Heaton, T., Kohler, M., Chandy, M., Guy, R. and Bunn, J., 2015. Community Seismic Network: A Dense Array to Sense Earthquake Strong Motion. Seismological Research Letters.

Couclelis, H., 1992. People manipulate objects (but cultivate fields): Beyond the raster-vector debate in gis. In: A. U. Frank, I. Campari and U. Formentini (eds), Theories and Methods of Spatio-Temporal Reasoning in Geographic Space: International Conference GIS - From Space to Territory: Theories and Methods of Spatio-Temporal Reasoning Pisa, Italy, September 21-23, 1992 Proceedings, Springer Berlin Heidelberg, Berlin, Heidelberg, pp. 65-77.

Galić, Z., 2016. Spatio-Temporal Data Streams. Springer New York.

GeoJSON, 2017. http://geojson.org/ (24 May 2017).

Grapenthin, R., Johanson, I. A. and Allen, R. M., 2014. Operational real-time GPS-enhanced earthquake early warning. Journal of Geophysical Research: Solid Earth 119(10), pp. 79447965.

Grapenthin, R., Johanson, I. and Allen, R., n.d. 32 G-larmS: Integrating Real-Time GPS into Earthquake Early Warning. Berkeley Seismological Laboratory p. 70.

Highcharts, 2017. https://www.highcharts.com/ (12 Apr. 2017).

Hudnut, K. W., Bock, Y., Galetzka, J. E., Webb, F. H. and Young, W. H., 2002. The Southern California Integrated GPS Network (SCIGN). Seismotechtonics in Convergent Plate Boundary (January), pp. 167-189.

Kemp, K., 1996. Fields as a framework for integrating GIS and environmental process models. Part 1: Representing spatial continuity. Transactions in GIS.

Kong, Q., Kwony, Y. W., Schreierz, L., Allen, S., Allen, R. and Strauss, J., 2015. Smartphone-based networks for earthquake detection. In: Innovations for Community Services (I4CS), 2015 15th International Conference on, pp. 1-8.

Liang, Q., Nittel, S. and Hahmann, T., 2016. From Data Streams to Fields: Extending Stream Data Models with Field Data Types. In: 9th International Conference on Geographic Information Science (GIScience), Montreal, Canada, September, Springer LNCS, pp. 1242-1253.
MongoDB, 2017. https://www.mongodb.com (24 May 2017).

Muthukrishnan, S., 2005. Data streams: Algorithms and applications. Foundations and Trends in Theoretical Computer Science 1(2), pp. 117-236.

Panizzi, E., 2016. The SeismoCloud App: Your Smartphone As a Seismometer. In: Proceedings of the International Working Conference on Advanced Visual Interfaces, AVI '16, ACM, New York, NY, USA, pp. 336-337.

Real-time Earthquake Monitoring Visualization for SCIGN Stations, University of Maine, 2017. http://shakeviz.geosensornetworks.net/ (18 July 2017).

Real-time Feeds and Notifications, 2017. https://earthquake.usgs.gov/earthquakes/feed/ (24 May 2017).

Socket.IO, 2017. https://socket.io (30 May 2017).

Stonebraker, M., Çetintemel, U. and Zdonik, S., 2005. The 8 requirements of real-time stream processing. ACM SIGMOD Record 34(4), pp. 42-47.

Wright, T. J., Houlié, N., Hildyard, M. and Iwabuchi, T., 2012. Real-time, reliable magnitudes for large earthquakes from $1 \mathrm{~Hz}$ GPS precise point positioning: The 2011 Tohoku-Oki (Japan) earthquake. Geophysical Research Letters 39(12), pp. n/a-n/a. 